of force in the plane which depend not only upon the position of the point but also upon the direction through the point. In this paper, the geometry of the dynamical trajectories of such generalized fields of force in space is studied. It results that the $\infty^{5}$ dynamical trajectories are completely characterized by two geometric properties as follows. For each of the $\infty^{1}$ curves of the $\infty^{5}$ trajectories which pass through a given lineal element $E$, construct the osculating plane and sphere at $E$. The two properties are: (I) The $\infty^{1}$ trajectories all have the same osculating plane, and (II) The locus of the centers of the osculating spheres is a straight line. Finally velocity systems are defined and shown to be curvature trajectories. (Received December 28, 1942.)

\title{
Statistics And Probability
}

\section{Henry Scheffé: On solutions of the Behrens-Fisher problem,} based on the t-distribution.

The problem is to find confidence intervals for the difference of the means of two normal populations when the ratio of their variances is unknown. Certain disadvantages inhere in solutions hitherto proposed: Some data are completely discarded when the sample sizes are unequal (Neyman), or the confidence coefficient is not known exactly (Fisher), or existing tables are inadequate if the confidence coefficient is to be 95 or 99 per cent (Wilks). The present solution is as follows: Let the samples be $\left(x_{1}, x_{2}, \cdots, x_{m}\right)$ and $\left(y_{1}, y_{2}, \cdots, y_{n}\right)$, where the $x$ 's and $y^{\prime}$ 's are mutually independently normally distributed, the former with mean $a_{x}$ and variance $\sigma_{x}^{2}$, the latter with mean $a_{y}$ and variance $\sigma_{y}^{2}$. Assume $m \leqq n$. Let $\bar{x}, \bar{y}$ be the sample means, and $\delta=a_{x}-a_{y}$. Then $(\bar{x}-\bar{y}-\delta) / Q^{1 / 2}$ has the Fisher $t$-distribution with $m-1$ degrees of freedom if $m(m-1) Q=\sum_{i=1}^{m}\left(u_{i}-\bar{u}\right)^{2}, u_{i}=x_{i}-(m / n)^{1 / 2} y_{i}, \bar{u}=\sum_{i=1}^{m} u_{i} / m$. This leads immediately to confidence intervals for $\delta$, and it is shown that of a certain class these have the minimum expected length. (Received January 7, 1943.)

\section{TOPOLOGY}

\section{M. G. Ettlinger: On irreducible continuous curves.}

It is proved that, in a connected space satisfying Axioms 0-2 of R. L. Moore's Foundations of point set theory (Amer. Math. Soc. Colloquium Publications, vol. 13, 1932), every compact and closed point set is a subset of a compact continuous curve, and every compact and closed point set having no continuum of condensation is a subset of a compact hereditary continuous curve. It is shown that if, in a space satisfying Axioms $0-1, M$ is a locally compact continuous curve which is an irreducible continuum about a closed subset $T$ of $M$, then every continuum of condensation of $M$ is a continuum of condensation of $T$. It is then demonstrated that in a connected space satisfying Axioms 0-2, every compact and closed point set having no continuum of condensation is a subset of a compact continuous curve having no continuum of condensation. (Received January 21, 1943.)

\section{O. G. Harrold: A decomposition theorem for certain compacta.}

Let $\psi_{n}$ denote the property of being locally connected in dimension $n$ in the sense of homotopy $(n-L C)$ at a point $p$ of a compactum $X$. Let $\Delta_{n}$ denote the $\psi_{n}$-singular points of $X$. Let $\mathfrak{S}_{n}^{n+1}$ denote the class of compacta which are $L C^{n}(i-L C$, $i=0,1, \cdots, n)$ and such that small singular $(n+1)$-spheres bound. If $X \in \mathfrak{C}_{n}^{n+1}$, either $\bar{\Delta}_{n+1}$ is vacuous or contains nondegenerate connected sets. Also, analogous to 
results of R. L. Wilder (Decompositions of compact metric spaces, Amer. J. Math. vol. 63 (1941) pp. 691-697), if $X \in \mathbb{C}_{n}^{n+1}$ and the $\psi_{n+1}$-prime parts of $X$ are simply-iconnected, $i=1,2, \cdots, n+1$, then the space $X^{\prime}$ of $\psi_{n+1}$-prime parts of $X$ is $L C^{n+1}$. For $n \geqq 1$, by use of a well known theorem due to Hurewicz, these theorems may be stated in terms of Vietoris cycles with integer coefficients. (Received January 30, 1943.)

\section{A. H. Stone: Connectedness and coherence.}

This paper is concerned with the incidence geometry of a topological space $S$, that is, the relations between the numbers of components of sets and their frontiers, unions and intersections, expressed in terms of the zero-dimensional Betti number $b_{0}$ and the coherence $c=r+1$ (Eilenberg, Fund. Math. vol. 27 (1936)). Among others, the following theorems are obtained. The coherence of a space may be defined using open sets instead of closed ones. For any set $A, b_{0}(\mathcal{F}(A)) \leqq b_{0}(\bar{A})+b_{0} \overline{(\mathcal{C}(A))}+c(S)-1$, and $\sum b_{0}(\mathcal{F}(C)) \leqq b_{0}(\bar{A})+c(S)-1$, the summation extending over the components $\dot{C}$ of the complement of $A$. A theorem of Eilenberg on coverings of order 3 is extended and sharpened, yielding the corollary that if $S$ is unicoherent and $f(A) \cap \mathcal{f}(B) \subset A \cap B \cap J(A \cup B)$ then $b_{0}(A \cap B)+b_{0}(A \cup B)=b_{0}(A)+b_{0}(B)$. Several other theorems, as well as extensions of some known results, are obtained for unicoherent spaces, and some of them are extended to the multicoherent case. Most of the theorems apply to sets which need not be open or closed. $S$ is assumed to be normal; but if $S$ is unicoherent, methods are developed which dispense completely with separation axioms. Applications are made to spaces of coherence 2, and to euclidean space. (Received January 20, 1943.) 